\title{
Multiscale, Generalised Stochastic Solute Transport Model in One Dimension
}

\subsection{Introduction}

In Chapter 3 and 4, we have developed a stochastic solute transport model in 1-D without rosorting to simplifying Fickian assumptions, but by using the idea that the fluctuations in velocity are influenced by the nature of porous medium. We model these fluctuations through the velocity covariance kernel. We have also estimated the dispersivity by taking the realisations of the solution of the SSTM and using them as the observations in the stochastic inverse method (SIM) based on the maximum likelihood estimation procedure for the stochastic partial differential equation obtained by adding a noise term to the advectiondispersion equation. We have confined the estimation of dispersitivities to a flow length of 1 $\mathrm{m}$ (i.e, $x \in[0,1]$ ) except in Chapter 3, section 3.10, where we have estimated the dispersitivities up to $10 \mathrm{~km}$ using the SIM by simplifying the SSTM. This approach was proven to be computationally expensive and the approximation of the SSTM we have developed was based on the spatial average of the variance of the fluctuation term over the flow length. Further, the solution is based on a specific kernel. This development in Chapter 3 is inadequate to examine the scale dependence of the dispersitivity. Therefore, we set out to develop a dimensionless model for any given arbitrary flow length, $L$, in this Chapter for any given velocity kernel provided that we have the eigen functions in the form given by equation (4.2.3). Then we examine the dispersivities in relation to the flow lengths to understand the multi-scale behaviour of the SSTM.

The starting point of the development of the multi-scale SSTM is the Langevin equation for the SSTM, which is interpreted locally. From equation (4.9.1), the Langevin equation can be written as,

$$
d C_{x}(t)=-\alpha_{x}\left(C_{x}(t), \bar{V}(x, t), x\right) d t+\beta_{x}\left(C_{x}(t), \frac{\partial C_{x}}{\partial x}, \frac{\partial^{2} C_{x}}{\partial x^{2}}, x\right) d w(t)
$$

where the coefficients $\alpha_{x}$ and $\beta_{x}$ are dependent on $x, C_{x}(t)$ and $\bar{V}(x, t)$; and $C_{x}(t), \frac{\partial C_{x}}{\partial x}, \frac{\partial^{2} C_{x}}{\partial x^{2}}$ and $x$, respectively. $d w(t)$ are the standard Wiener increments with zero-mean and $d t$ variance. As discussed in Chapter 4, equation (6.1.1) has to be interpreted carefully to understand it better. Equation (6.1.1) is a SDE and also an Ito diffusion with the coefficients depending on the functions of space variables. It gives us the time evolution of the concentration of solute at a given point $x$ which is denoted by subscript $x$. Obviously, the computation of $C_{x}$ also depends on how the spatial 
derivatives of $C_{x}$ are calculated. In that sense, equation (6.1.1) is a stochastic partial differential equation as the coefficients are functions of random quantities. But we avoid solving a SPDE by treating equation (6.1.1) as a SDE and interpreting it as an Ito integral which makes us to evaluate coefficients at the previous time point with respect to the current point of evaluation.

For simplicity, we will denote the coefficients as $\alpha_{x}$ and $\beta_{x}$. In Chapter 4 , we have derived explicit function for $\alpha_{x}$ and $\beta_{x}$ :

$$
\alpha=C_{x}(t) F_{x, 0}+\left(\frac{\partial C}{\partial x}\right)_{x} F_{x, 1}+\left(\frac{\partial^{2} C}{\partial x^{2}}\right)_{x} F_{x, 2} .
$$

Where

$$
\begin{gathered}
F_{x, 0}=\frac{\partial \bar{V}(x, t)}{\partial x}+\frac{h_{x}}{2} \frac{\partial^{2} \bar{V}(x, t)}{\partial x^{2}}, \\
F_{x, 1}=\bar{V}(x, t)+h_{x} \frac{\partial \bar{V}(x, t)}{\partial x}
\end{gathered}
$$

and,

$$
\begin{gathered}
F_{x, 3}=\frac{h_{x}}{2} \bar{V}(x, t) ; \\
\beta_{x}=\left(\beta_{0}^{2}+\beta_{1}^{2}+\beta_{2}^{2}\right)^{1 / 2},
\end{gathered}
$$

where,

$$
\begin{aligned}
& \beta_{0}=C_{x}(t) \sqrt{a_{00}}, \\
& \beta_{1}=\left(\frac{\partial C}{\partial x}\right)_{x} \sqrt{a_{11}}, \\
& \beta_{2}=\left(\frac{\partial^{2} C}{\partial x^{2}}\right)_{x} \sqrt{a_{22}},
\end{aligned}
$$

and

$$
a_{i i}=\sigma^{2} \sum_{j=1}^{m} \lambda_{j} P_{i j}^{2}, \quad(i, 0,1,2)
$$

In equation (6.1.10), $\sigma^{2}$ is the variance of the covariance kernel, $\lambda_{j}$ are eigen functions, and for the domain of $x \in[0,1]$, 


$$
\begin{aligned}
P_{0 j}(x)= & {\left[g_{i j}-2 \sum_{k=2}^{p_{j}} g_{k j} r_{k j}\left(x-s_{k j}\right) e^{-r_{k j}\left(x-s_{k j}\right)^{2}}\right] } \\
& +\left(\frac{h_{x}}{2}\right)\left[4 \sum_{k=2}^{p_{j}} g_{k j} r_{k j}^{2}\left(x-s_{k j}\right)^{2} e^{-r_{k j}\left(x-s_{k j}\right)^{2}}-2 \sum_{k=2}^{p_{j}} g_{k j} r_{k j} e^{-r_{k j}\left(x-s_{k j}\right)^{2}}\right], \\
P_{1 j}(x)= & g_{0 j}+g_{1 j} x+\sum_{k=2}^{p_{j}} g_{k j} e^{-r_{j j}\left(x-s_{k j}\right)^{2}} \\
& +\left(\frac{h_{x}}{2}\right)\left[2\left(g_{i j}-2 \sum_{k=2}^{p_{j}} g_{k j} r_{k j}\left(x-s_{k j}\right) e^{-r_{k j}\left(x-s_{k j}\right)^{2}}\right)\right],
\end{aligned}
$$

and

$$
P_{2 j}(x)=\left(\frac{h_{x}}{2}\right)\left[g_{0 j}+g_{1 j} x+\sum_{k=2}^{p_{j}} g_{k j} e^{-r_{k j}\left(x-s_{k j}\right)^{2}}\right] .
$$

Equation (6.1.1) to (6.1.13) constitute the Langevin form of the SSTM. It should be noted that the functions $P_{i j}$ are only valid for $x \in[0,1]$. If we normalize the spatial variable $x$ to remain with in $[0,1]$, then we can use the results in Chapter 4 to obtain $P_{i j}$. We develop the dimensionless Langevin form of the SSTM in section 6.2.

One should note that the Langevin equation for any system reflect the role of external noise to the system under consideration (van Kampen, 1992). Even though we have derived equation (6.1.1) starting from the mass conservation of solute particles, the fluctuations associate with hydrodynamics dispersion are a result of dissipation of energy of particles due to momentum changes associated near to the surfaces of porous medium. For $\alpha$ physical ensemble of solute particles, porous medium through which it flows act as an external source of noise. From this point of review, the Langevin type equation for solute concentration is justified. As a SDE, equation (6.1.1) is a Wiener process with stochastic, at best nonlinear, time-dependent coefficients, and it is also an Ito diffusion which should be interpreted locally, i.e., for a given $x$ and $t$, equation (6.1.1) is valid only for short time intervals beyond $t$. This naturally leads us to evaluate the associated spatial derivatives at the previous time, which is valid according to Ito's interpretation of stochastic integral. In terms of discretized times, $t_{0}, t_{1}, \ldots, t_{i}, t_{i+1}, \ldots$, equation (6.1.1) can be written as,

$$
\begin{aligned}
d C_{x}(t)=C_{x}(t+1)-C_{x}(t) & =\alpha_{x}\left(C_{x}\left(t_{i}\right), \bar{V}\left(x, t_{i}\right),\left(\frac{\partial \bar{V}}{\partial x}\right)_{t_{i}},\left(\frac{\partial^{2} \bar{V}}{\partial x^{2}}\right)_{t_{i}}\right)\left(t_{i+1}-t_{i}\right) \\
& +\left(\beta_{x}\right)\left(C_{x}\left(t_{i}\right), \bar{V}\left(x, t_{i}\right),\left(\frac{\partial \bar{V}}{\partial x}\right)_{t_{i}},\left(\frac{\partial^{2} \bar{V}}{\partial x^{2}}\right)_{t_{i}}\right) d \omega\left(t_{i}\right)
\end{aligned}
$$

where the drift coefficient, $\alpha_{x}$, and the diffusion coefficient, $\beta_{x}$, are evaluated at time $=t_{i}$. This restrictive nature of equation (6.1.14) in evaluating the coefficient has to be taken in to account in developing numerical algorithms to solve it. 


\subsection{Partially Dimensionless SSTM with Flow Length $L$}

We start the derivation of partially dimensionless SSTM by defining the dimensionless distance, $Z$, as:

$$
\mathrm{Z}=x / L
$$

where $L$ is the total flow length.

When $x \in[0, L], \quad Z \in[0,1]$.

If $C_{0}$ is a constant concentration defined such a way that $C_{0}=$ maximum of $C_{x}(t)$ for all $x$ and $t$, then $C_{0} \geq C_{x}(t)$ for any $t$ and $x$. We can define dimensional concentration $\Gamma(t)$ as,

$$
\Gamma(t)=\frac{C_{x}}{C_{0}}
$$

From equation (6.2.1),

$$
\begin{gathered}
\frac{\partial Z}{\partial x}=\frac{1}{L} \\
\frac{\partial C_{x}}{\partial x}=\frac{\partial\left(C_{0} \Gamma\right)}{\partial Z} \cdot \frac{\partial Z}{\partial x}=\frac{C_{0}}{L} \frac{\partial \Gamma}{\partial Z} \\
\frac{\partial^{2} C_{x}}{\partial x^{2}}=\frac{\partial}{\partial x}\left(\frac{\partial C_{x}}{\partial x}\right)=\frac{\partial}{\partial x}\left(\frac{C_{0}}{L} \frac{\partial \Gamma}{\partial Z}\right)=\frac{\partial}{\partial Z}\left(\frac{C_{0}}{L} \frac{\partial \Gamma}{\partial Z}\right) \cdot \frac{\partial Z}{\partial x}=\frac{C_{0}}{L^{2}} \frac{\partial^{2} \Gamma}{\partial Z^{2}} .
\end{gathered}
$$

As the domain of $x$ is the generalized SSTM is from 0 to 1 , we can replace $x$ with $Z$ in the dimensionless generalized SSTM. For example, $F_{x, 0}$ becomes $F_{z, 0}$.

$$
\begin{aligned}
F_{Z, 0} & =\frac{\partial \bar{V}(Z, t)}{\partial x}+\frac{h_{\mathrm{Z}}}{2} \frac{\partial^{2} \bar{V}(\mathrm{Z}, t)}{\partial x^{2}}=\frac{\partial \bar{V}(\mathrm{Z}, t)}{\partial \mathrm{Z}} \cdot \frac{\partial \mathrm{Z}}{\partial x}+\frac{h_{\mathrm{Z}}}{2} \frac{\partial}{\partial x}\left(\frac{\partial \bar{V}}{\partial Z} \cdot \frac{\partial \mathrm{Z}}{\partial x}\right) \\
& =\frac{1}{L} \frac{\partial \bar{V}}{\partial \mathrm{Z}}+\frac{h_{\mathrm{Z}}}{2 L} \frac{\partial}{\partial \mathrm{Z}}\left(\frac{\partial \bar{V}}{\partial Z}\right)\left(\frac{\partial Z}{\partial x}\right)=\frac{1}{L} \frac{\partial \bar{V}}{\partial \mathrm{Z}}+\frac{h_{\mathrm{Z}}}{2 L^{2}} \frac{\partial^{2} \bar{V}}{\partial \mathrm{Z}^{2}} .
\end{aligned}
$$

Similarly,

$$
\begin{gathered}
F_{\mathrm{Z}, 1}=\bar{V}(\mathrm{Z}, t)+\frac{h_{\mathrm{Z}}}{L} \frac{\partial \bar{V}}{\partial \mathrm{Z}} ; \text { and } \\
F_{\mathrm{Z}, 2}=\frac{h_{\mathrm{Z}}}{2} \bar{V}(\mathrm{Z}, t) .
\end{gathered}
$$

$P_{0 j}(\mathrm{Z}), P_{1 j}(\mathrm{Z})$ and $P_{2 j}(\mathrm{Z})$ are obtained by simply replacing $x$ in $P_{0 j}(x), P_{1 j}(x)$ and $P_{2 j}(x)$ expressions by $Z$, because these expressions are derived for $[0,1]$ domain. 
Similarly,

$$
\begin{gathered}
\beta_{0}(Z)=C_{0} \Gamma(t) \sqrt{a_{00}(Z)} \\
\beta_{1}(Z)=\frac{C_{0}}{L} \frac{\partial \Gamma}{\partial Z} \sqrt{a_{11}(Z)}, \text { and } \\
\beta_{2}(Z)=\frac{C_{0}}{L^{2}} \frac{\partial^{2} \Gamma}{\partial Z^{2}} \sqrt{a_{22}(Z)}
\end{gathered}
$$

Now we can write equation (6.1.1) in the following manner:

$$
\begin{gathered}
d\left(C_{0} \Gamma\right)=-\alpha_{Z}(Z) d t+\beta_{Z}(Z) d \omega(t), \\
d \Gamma=\frac{-\alpha Z(Z)}{C_{0}} d t+\frac{\beta_{Z}(Z)}{C_{0}} d \omega(t) .
\end{gathered}
$$

where

$$
\begin{gathered}
\frac{\alpha_{Z}(\mathrm{Z})}{C_{0}}=\Gamma F_{\mathrm{Z}, 0}+\frac{1}{L} \frac{\partial \Gamma}{\partial Z} F_{\mathrm{Z}, 1}+\frac{1}{L^{2}} \frac{\partial^{2} \Gamma}{\partial \mathrm{Z}^{2}} F_{\mathrm{Z}, 2} \\
\frac{\beta_{\mathrm{Z}}(\mathrm{Z})}{C_{0}}=\left\{\left(\Gamma^{2} a_{00}(\mathrm{Z})\right)+\frac{1}{L^{2}}\left(\frac{\partial \Gamma}{\partial Z}\right)^{2} a_{11}(\mathrm{Z})+\frac{1}{L^{4}}\left(\frac{\partial^{2} \Gamma}{\partial \mathrm{Z}^{2}}\right)^{2} a_{22}(\mathrm{Z})\right\}^{1 / 2}
\end{gathered}
$$

Therefore, the Langevin form of the generalized SSTM is given by

$$
d \Gamma=-\alpha_{\mathrm{Z}} d t+\beta_{\mathrm{Z}} d \omega(t), 0 \leq \mathrm{Z} \leq 1
$$

where $\bar{\alpha}_{Z}=\frac{\alpha_{Z}(Z)}{C_{0}}$ and $\bar{\beta}_{Z}=\frac{\beta_{Z}(Z)}{C_{0}}$.

Using equation (6.2.14) we can compute the time course of the dimensionless concentration for any given $L$.

The dimensionless/concentration, $\Gamma$, varies from 0 to $1 . C_{Z}(t)$ is proportioned to the number of solute moles within a unit volume of porous/water matrix, and $C_{0}$ is proportional to the maximum possible number of solute moles within the same matrix. Therefore, $\Gamma=C_{Z}(t) / C_{0}$ can be interpreted as the likelihood (probability) of finding solute moles within the matrix.

It should be noted that time, $t$, is not a dimensionless quantity and therefore, equation (6.2.14) is partially dimensionless equation. We will explore the dispersivity using equation (6.2.14) first before discussing a completely dimensionless equation. 


\subsection{Computational Exploration of the Langevin form of SSTM}

Equation (6.2.14) is not only an expression of how the solute disperses within a porous media but also an expression of nature of dispersion. Being a SDE, the drift coefficient $\left(\bar{\alpha}_{z}\right)$ portrays the dispersion due to the convective forces and the diffusive coefficient $\left(\bar{\beta}_{Z}\right)$ shows the dynamical behaviour of hydrodynamic dispersion. As $Z$ has the range from 0 to 1 in equation (6.2.14), we can compute $\alpha_{Z}$ and $\beta_{Z}$ values for a specific $Z$ value and examine how they change over time. (We use $C_{0}=1.0$ for computations, and therefore, $\bar{\alpha}_{Z}=\alpha_{Z}$ and $\bar{\beta}_{Z}=\beta_{Z}$.) We have developed a finite difference algorithm to compute $\alpha_{Z}$ and $\beta_{Z}$ adhering to the Ito integration as we have done before. Figure $6.1 \mathrm{a}$ and $6.1 \mathrm{~b}$ show the time courses of $-\alpha_{Z}$ and $\beta_{\mathrm{Z}}$ at $Z=0.5$, respectively, for different $\sigma^{2}$ values when $L=1 m$ (All times are given in days and $b=0.1$. At low $\sigma^{2}$ values, $-\alpha_{Z}$ behaves almost as a smooth deterministic function but at high $\sigma^{2}$ values it shows irregular behaviours. In these calculations, we have kept the mean velocity $\bar{V}$ at a constant value (0.5), therefore only fluctuating component affecting $\alpha_{\mathrm{Z}}$ function is the solute concentration and its spatial derivatives. Further, Figure 6.1a and $6.1 \mathrm{~b}$ only show a single realization for each $\sigma^{2}$ values. When we explore multiple realizations (not shown here), we see that randomness of $\alpha_{Z}$ and $\beta_{Z}$ increases with higher $\sigma^{2}$. One distinct feature of Figure $6.1 \mathrm{~b}$ for $\beta_{\mathrm{Z}}$ is that $\beta_{\mathrm{Z}}$ is almost negligible for very small values of $\sigma^{2}$ but increases quite sharply for higher $\sigma^{2}$ values. $\alpha_{z}$ does not behave in this manner. However, we can not ignore the effect of $\sigma^{2}$ at low values in computing $\Gamma(Z)$, which has a follow-on affect on subsequent calculation. In other words, the affects of porous media, which $\sigma^{2}$ and the covariance kernel signify, can not be ignored as they affect the flow velocities significantly in making them stochastic. Figure $6.2 \mathrm{a}$ and $6.2 \mathrm{~b}$ show $-\alpha_{z}$ and $\beta_{\mathrm{Z}}$ realization at $Z=0.5$ when $L=5 \mathrm{~m}$. The behaviours of $-\alpha_{Z}$ and $\beta_{Z}$ realizations are similar to those shown in Figures 6.1a and 6.1b. Figure $6.3 \mathrm{a}$ and Figure $6.3 \mathrm{~b}$ show the similar trends for $L=10 \mathrm{~m}$. It should be noted that as $L$ is increased, the time duration for the numerical solution of equation (6.2.14) should be increased. For example, when $L=10 \mathrm{~m}$, the model was run for 25 days to obtain Figures 6.3a and 6.3b. However, the order of magnitude for $\alpha_{Z}$ and $\beta_{Z}$ has not changed as we change $L$ in an order of magnitude. 

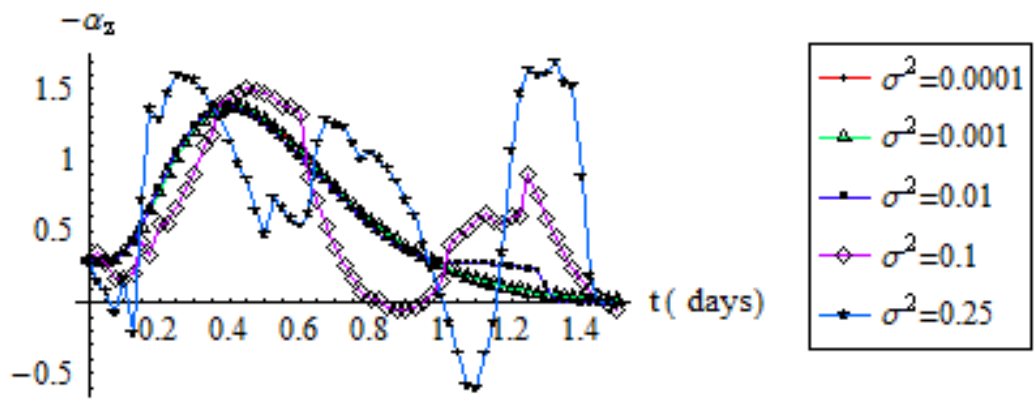

(a)
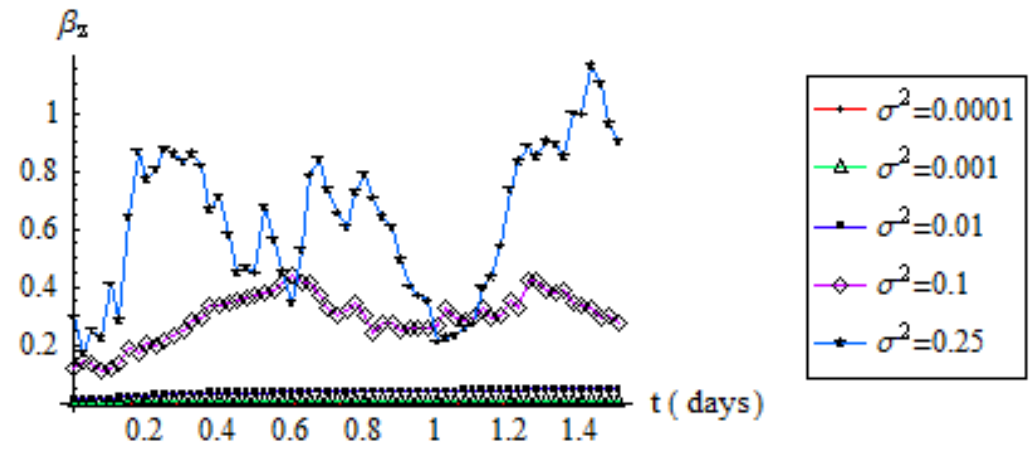

(b)

Figure 6.1. (a) Realizations of $-\alpha_{Z}$ at $Z=0.5$ when $L=1 m, b=0.1$ and $\bar{V}=0.5 \mathrm{~m} /$ day for different $\sigma^{2}$ values; (b) Realizations of $\beta_{Z}$ at $Z=0.5$ when $L=1 \mathrm{~m}$, $b=0.1$ and $\bar{V}=0.5 \mathrm{~m} /$ day for different $\sigma^{2}$ values.
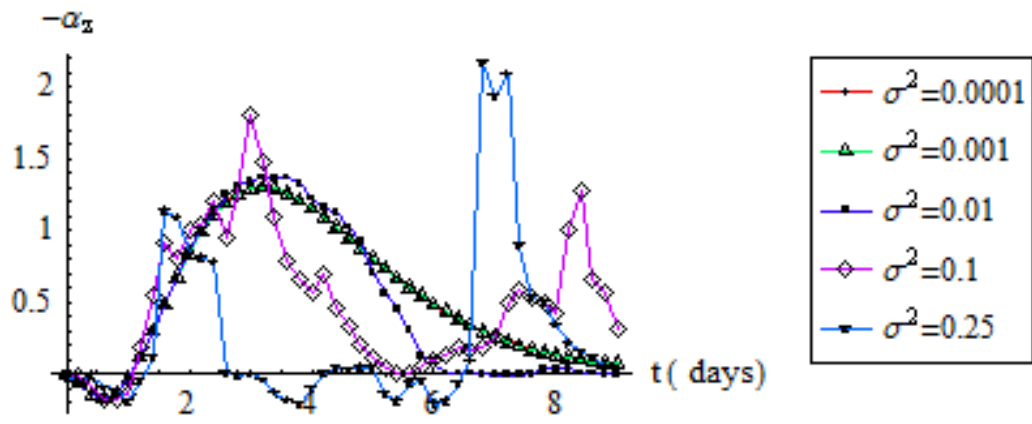

(a) 

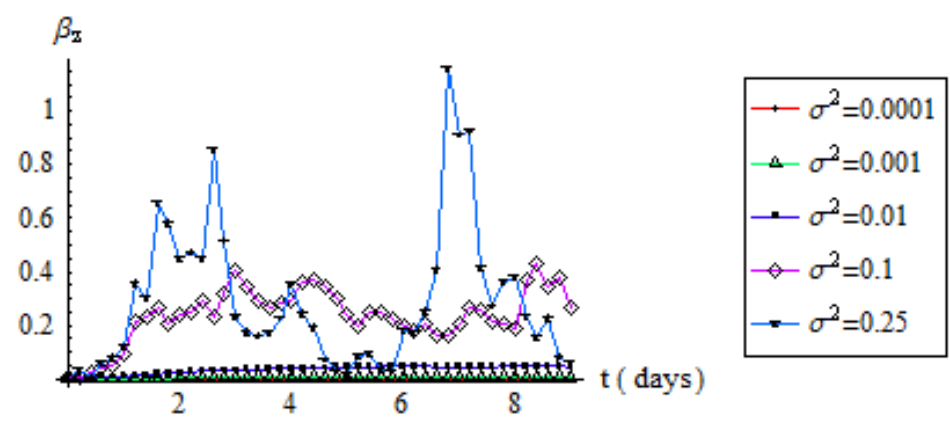

(b)

Figure 6.2. (a) Realizations of $-\alpha_{Z}$ at $Z=0.5$ when $L=5 m, b=0.1$ and $\bar{V}=0.5 \mathrm{~m} /$ day for different $\sigma^{2}$ values; (b) Realizations of $\beta_{Z}$ at $Z=0.5$ when $L=5 \mathrm{~m}$, $b=0.1$ and $\bar{V}=0.5 \mathrm{~m} /$ day for different $\sigma^{2}$ values.
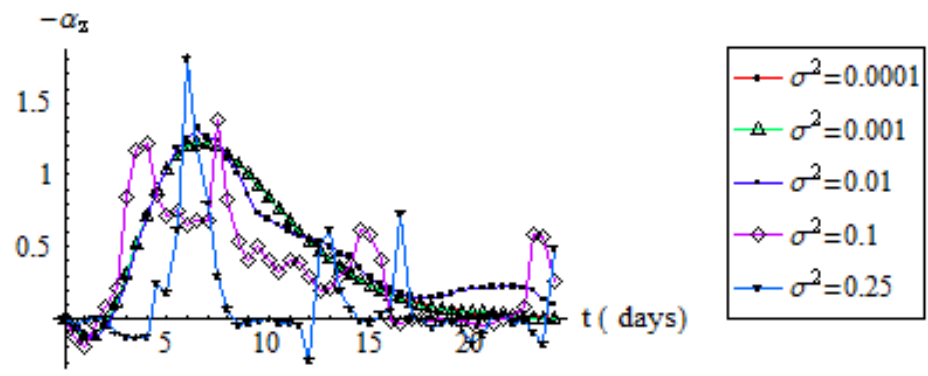

(a)
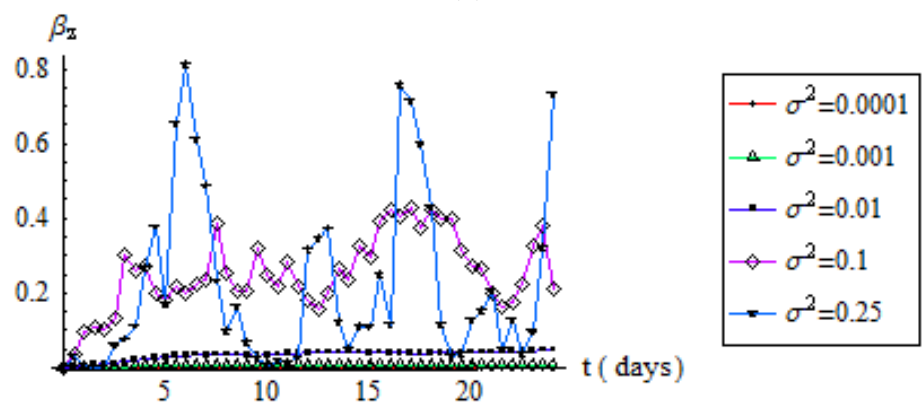

(b)

Figure 6.3. (a) Realizations of $-\alpha_{Z}$ at $Z=0.5$ when $L=10 \mathrm{~m}, \quad b=0.1$ and $\bar{V}=0.5 \mathrm{~m} /$ day for different $\sigma^{2}$ values; (b) Realizations of $\beta_{Z}$ at $Z=0.5$ when $L=10 \mathrm{~m}, b=0.1$ and $\bar{V}=0.5 \mathrm{~m} /$ day for different $\sigma^{2}$ values. 


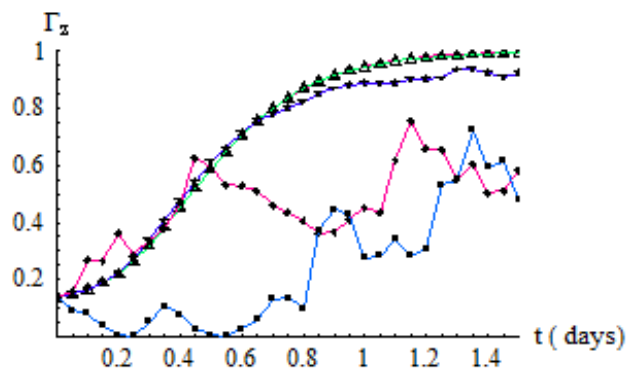

$$
\begin{aligned}
& -\sigma^{2}=0.0001 \\
& -\sigma^{2}=0.001 \\
& -\sigma^{2}=0.01 \\
& -\sigma^{2}=0.1 \\
& -\sigma^{2}=0.25
\end{aligned}
$$

(a)

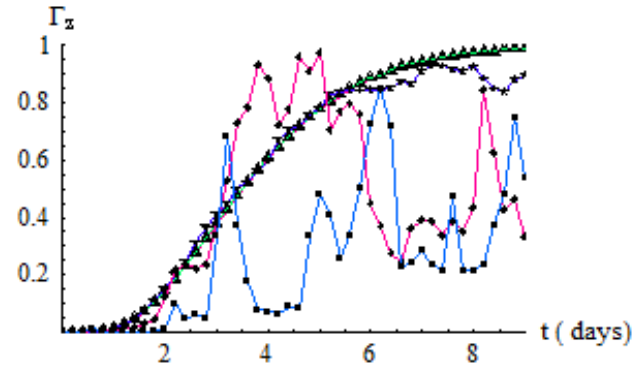

$$
\begin{aligned}
& -\sigma^{2}=0.0001 \\
& \triangle \sigma^{2}=0.001 \\
& -\sigma^{2}=0.01 \\
& \rightarrow \sigma^{2}=0.1 \\
& -\sigma^{2}=0.25
\end{aligned}
$$

(b)

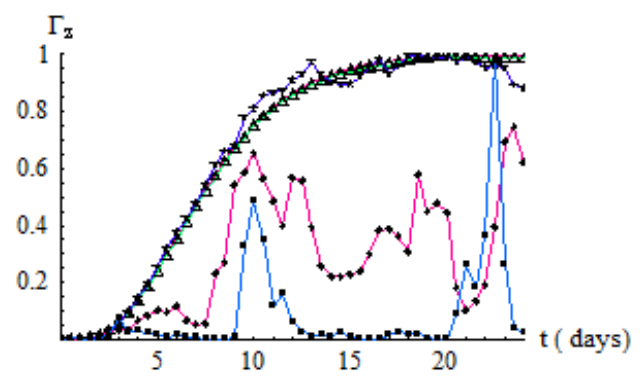

$$
\begin{aligned}
& -\sigma^{2}=0.0001 \\
& \triangle \sigma^{2}=0.001 \\
& -\sigma^{2}=0.01 \\
& \leftarrow \sigma^{2}=0.1 \\
& -\sigma^{2}=0.25
\end{aligned}
$$

(c)

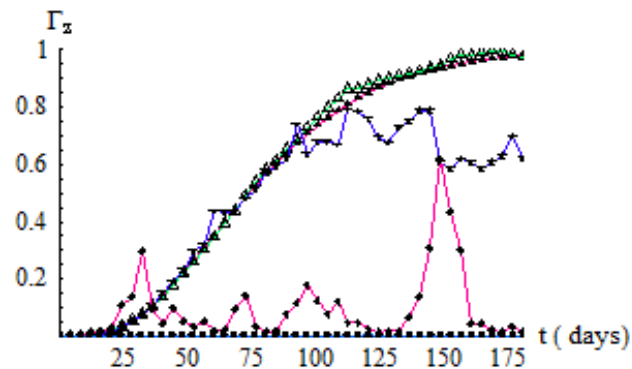

$$
\begin{aligned}
& -\sigma^{2}=0.0001 \\
& \triangle \sigma^{2}=0.001 \\
& -\sigma^{2}=0.01 \\
& \because \sigma^{2}=0.1 \\
& -\sigma^{2}=0.25
\end{aligned}
$$

(d)

Figure 6.4. Realizations of $\Gamma_{Z}$ at $Z=0.5$ when $b=0.1, \bar{V}=0.5$ for (a) $L=1$, (b) $L=5$, (c) $L=10$ and (d) $L=100$ 
Figure $6.4 \mathrm{a}, 6.4 \mathrm{~b}, 6.4 \mathrm{c}$, and $6.4 \mathrm{~d}$ show the realization of $\Gamma(Z)$ at $Z=0.5$ when $L=1,5,10$, and 100, respectively, for different values of $\sigma^{2}$. (For all the calculations, we have used $b=0.1$ ). When $L=100 \mathrm{~m}$, we computed $\Gamma(Z)$ values for 175 days and the affects of $\sigma^{2}$ on $\Gamma(Z)$ is quite dramatic, and this shows that equation (6.2.14) can display very complex behaviour patterns albeit its simplicity. It should be noted however that $\sigma^{2}$ plays major role in delimiting the nature of realizations; $\sigma^{2}$ values high than 0.25 in these situations produces highly irregular concentration realizations which could occur in highly heterogeneous porous formations such as fractured formations.

\subsection{Dispersivities Based on the Langevin Form of SSTM for $L \leq 10 \mathbf{m}$}

One of the advantages of the partially dimensionless Langevin equation for the SSTM (equation 6.2.14) is that we can use it to compute the solute concentration profiles when the travel length $(L)$ is large. Equation (6.2.14) allows us to compute the dispersitivities using the stochastic inverse method (SIM) by estimating dispersivity for each realization of $\Gamma(Z)$. For the SIM, we need to modify the deterministic-advection and dispersion equation into a partially dimensionless one. We start with the deterministic advection-dispersion equation with additive Gaussian noise,

$$
\frac{\partial C}{\partial t}=D_{L} \frac{\partial^{2} C}{\partial x^{2}}-V_{x} \frac{\partial C}{\partial x}+\xi(x, t)
$$

where $D_{L}$ is the dispersion coefficient (dispersivity $\times V_{x}$ ).

The partially dimensionless form of equation (6.4.1) is,

$$
\frac{\partial \Gamma}{\partial t}=\frac{D_{L}}{L^{2}} \frac{\partial^{2} \Gamma}{\partial Z^{2}}-\frac{V_{x}}{L} \frac{\partial \Gamma}{\partial Z}+\xi(Z, t)
$$

where $\frac{D_{L}}{L^{2}}$ is now estimated using SIM when $V_{x}$ is known. Then the dispersivity value is (estimated $\left.\frac{D_{L}}{L^{2}}\right) \times L^{2} / V_{x}$.

Figure 6.5 show the scatter plots of dispersivity values estimated using the SIM for $L=1,5$, and $10 \mathrm{~m}$. Each plot in Figure 6.5 gives 30 estimates of the dispersivity for a given value $\sigma^{2}$. $\Gamma(Z)$ realizations were computed at $Z=0.5$ and $b=0.1$ for all plots. Table 6.1 summarizes the results giving the mean of each plot. We will compare these results with available data for dispersivities later in this chapter. 

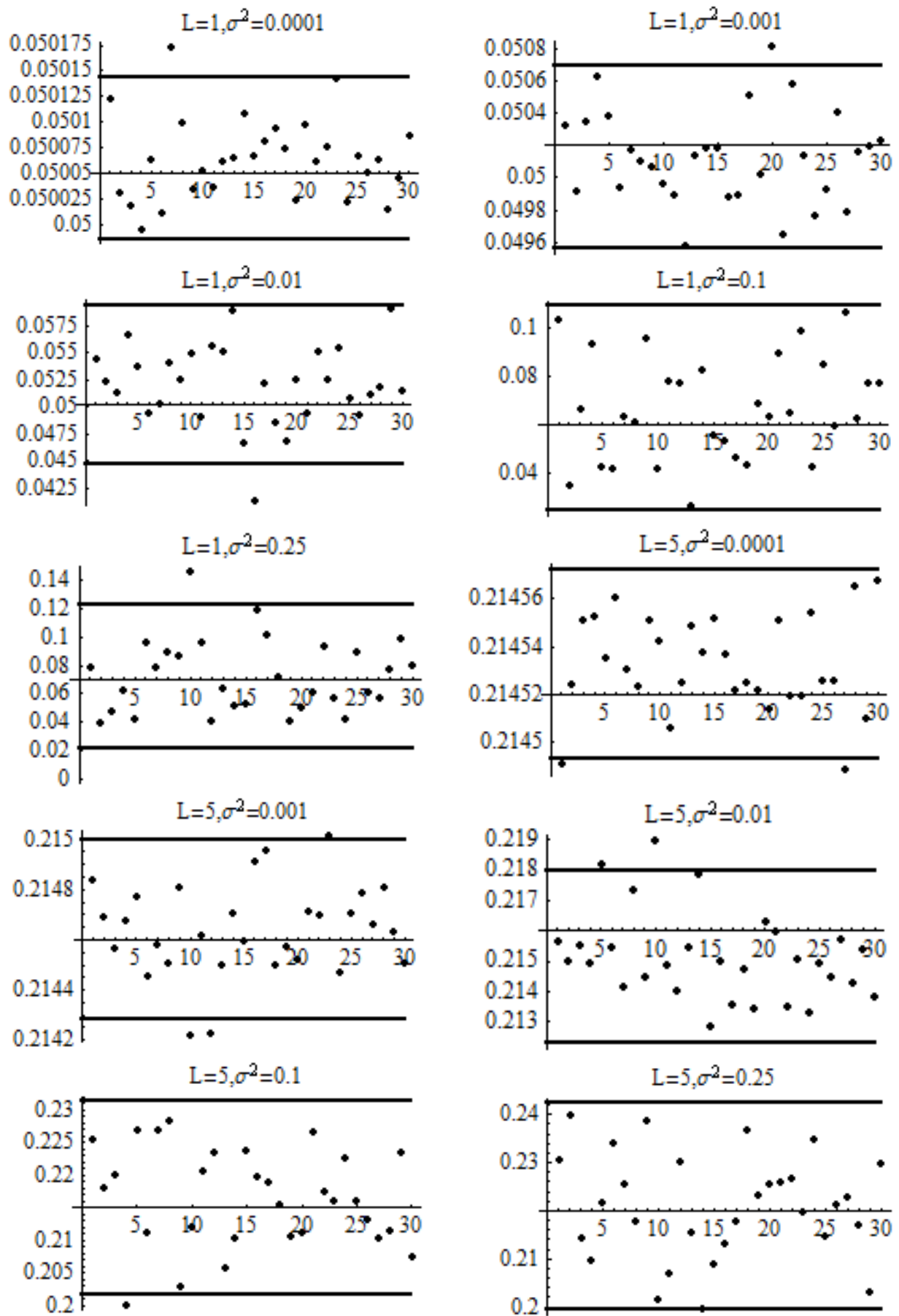

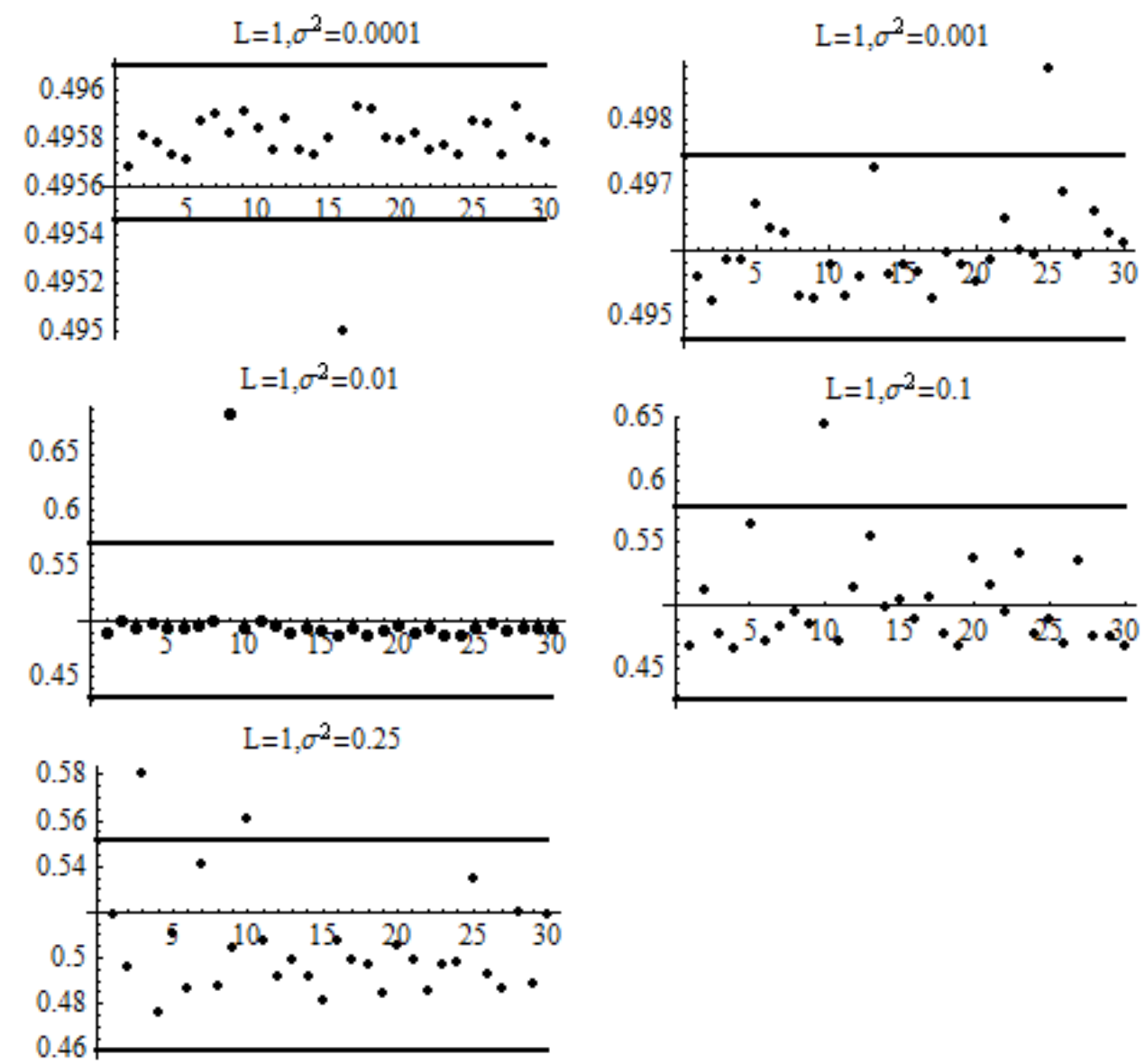

Figure 6.5. Dispersivities estimated from SSTM for $L=1,5$, and 10 .

\begin{tabular}{cccc}
\hline$\sigma^{2}$ & \multicolumn{3}{c}{ Dispersivity } \\
\cline { 2 - 4 } & $\mathrm{L}=1$ & $\mathrm{~L}=5$ & $\mathrm{~L}=10$ \\
\hline 0.0001 & 0.050064 & 0.050112 & 0.0495545 \\
0.001 & 0.0501232 & 0.05082 & 0.0511587 \\
0.01 & 0.0520638 & 0.0604215 & 0.0778382 \\
0.1 & 0.0669766 & 0.0832735 & 0.11899 \\
0.25 & 0.0723413 & 0.111142 & 0.253195 \\
0.4 & 0.0783754 & 0.142422 & 0.354335 \\
0.6 & 0.0843219 & 0.170975 & 0.427603 \\
0.8 & 0.0962623 & 0.225344 & 0.549473 \\
1 & 0.110849 & 0.256348 & 0.609508 \\
\hline
\end{tabular}

Table 6.1. Mean dispersivities for the data in Figure 6.5. 
As mentioned previously, the partially dimensionless equation (6.2.14) still requires us to compute for a large number of days when $L$ is large. While the computational times are still manageable, we would like to develop a completely dimensionless Langevin equation for the SSTM. This could be especially useful and insightful when the mean velocity $\bar{V}$ could be considered as a constant.

\subsection{Dimensionless Time}

We introduce dimensionless time, $\theta$, as,

$$
\theta=\bar{V}(Z, t) \cdot \frac{t}{L}
$$

where, $\bar{V}(Z, t)$ is mean velocity when $0 \leq Z \leq 1$, (m/day); $L$ is travel length, $\mathrm{m}$; and $t$ is time in days.

Therefore, if $\bar{V}=0.5, L=100$ and $0 \leq t \leq 200$, then, $0 \leq \theta \leq 1.0$. This allows us to compute $\Gamma(Z)$ realization for larger times.

Equation (6.2.14) can be written as,

$$
\Gamma(Z)=-\alpha_{Z} d t+\beta_{z} d \omega(t) .
$$

We can now change $d t=\frac{L}{\bar{V}} d \theta$, and the variance of $d \omega(t)=\Delta t=\frac{L}{\bar{V}} \Delta \theta$.

Therefore,

$$
\Gamma(Z)=\frac{-\alpha_{Z} L}{\bar{V}} d \theta+\beta_{z} d \omega(t)
$$

where $d w(\theta) \sim \mathrm{N}\left(0, \frac{L}{\bar{V}} d \theta\right)$.

The completely dimentionless Langevin form of the SSTM is therefore,

$$
\Gamma(Z)=\alpha_{Z, \theta} d \theta+\beta_{z} d \omega(\theta)
$$

where $d \omega(\theta)$ are the Wiener increment with zero-mean and $\frac{L}{\bar{V}} d \theta$ variance, and

$$
\alpha_{Z, \theta}=\frac{-\alpha_{Z} L}{\bar{V}}
$$

To use equation (6.5.3), we need to choose $\Delta \theta$ and the range of $\theta$ appropriately. Ideally $\frac{L}{\bar{V}} d \theta<0.0001$ for the Ito integration to be accurate; therefore, we should have for maximum $\Delta \theta$ as $\frac{0.0001 \bar{V}}{L}$. Suppose $\bar{V}=0.5, L=1000$, then $\Delta \theta<\frac{10^{-4} \times 0.5}{1000}$, i.e, $\Delta \theta<5 \times 10^{-8}$. 
As we can see we may not gain much computational advantage with a completely dimensionless Langevin form of the SSTM.

\subsection{Estimation of Field Scale Dispersivities}

We have estimated the longitudinal dispersivities using SSTM for two different boundary conditions:

(A) $\Gamma_{Z}=1$ at $Z=0$ and for $t \geq 0$; and

(B) $\Gamma_{Z}=1$ at $Z=0$ and for $0 \leq t \leq t_{R}$; and $\Gamma_{Z}=0$ at $Z=0$ for $t>t_{R}$.

$t_{R}$ is taken to be $1 / 3$ of the total time $(T)$ of the computational experiment. Table 6.1 and 6.2 show the dispersivity values for the boundary conditions A and B, respectively, when $L \leq 10 \mathrm{~m}$ based on 100 realisations for each of the boundary condition.

\begin{tabular}{cccc}
\hline \multirow{2}{*}{$\sigma^{2}$} & \multicolumn{3}{c}{ Dispersivity } \\
\cline { 2 - 4 } & $\mathrm{L}=1$ & $\mathrm{~L}=5$ & $\mathrm{~L}=10$ \\
\hline 0.0001 & 0.050013 & 0.050013 & 0.049828 \\
0.001 & 0.050035 & 0.050223 & 0.050226 \\
0.01 & 0.050646 & 0.055152 & 0.06112 \\
0.1 & 0.055176 & 0.079403 & 0.136904 \\
0.25 & 0.068846 & 0.108899 & 0.257902 \\
0.4 & 0.083342 & 0.16346 & 0.333472 \\
0.6 & 0.093185 & 0.191919 & 0.334818 \\
0.8 & 0.109335 & 0.251033 & 0.54346 \\
1 & 0.129395 & 0.331389 & 0.613823 \\
\hline
\end{tabular}

Table 6.2. Longitudinal dispersivities (mean) for the boundary condition A.

The values in Table 6.1 and 6.2 are similar for the similar values of $\sigma^{2}$ and $L$ showing that (1) the SSTM procedure is robust in evaluating the dispersivities, and (2) the computed mean dispersivities do not depend on the boundary conditions, $\mathrm{A}$ and $\mathrm{B}$. In these calculations, we have $\bar{V}_{Z}=0.5 \mathrm{~m} /$ day.

We have also computed the dispersivities for larger scales up to 10,000 $\mathrm{m}$, and Table 6.3 gives the mean values for the range of $L$ from $1 \mathrm{~m}$ to $10^{4} \mathrm{~m}$ under the boundary condition A, and Table 6.4 gives the mean values for the range of $L$ from $1 \mathrm{~m}$ to $10^{8} \mathrm{~m}$ for the boundary condition B. All mean values are calculated based on different sets of 100 realisations for each boundary condition. Except for the smallest $\sigma^{2}$ values $(0.0001$ and 0.001 ), the dispersivities have similar mean values for both boundary conditions, A and B. Therefore, it is quite reasonable to compute the dispersivities only for the boundary condition A for larger values of $L$. We can also hypothesise that the dispersivities are independent of the boundary conditions used to solve the SSTM. We have tested the SSTM for different values of $t_{R}>(1 / 3) T$ when $L>10 \mathrm{~m}$. Figure 6.6 depicts the dispersivity plotted against $\sigma^{2}$ and $L$ in $\log 10$ scale, and $\log 10$ (Dispersivity) is a linear function of $\log 10(L)$ 
and $\log 10\left(\sigma^{2}\right)$ for the most parts of the Log10 (Dispersivity) surface. Figure 6.7 shows the linear relationship of $\log 10$ (Dispersivity) vs $\log 10(L)$ for different values of $\sigma^{2}$, and Figure 6.8 shows the same for $\log 10$ (Dispersivity) vs $\log 10\left(\sigma^{2}\right)$ for different values of $L$. The gradient of the graphs are the same except for lower values of $\sigma^{2}(0.0001)$ and lower values of $L$ (1 and 5). Therefore, we develop the following statistical nonlinear regression models for these significant relationships:

$$
\begin{gathered}
D_{s}=C_{1}\left(\sigma^{2}\right)^{m 1} \text {, and } \\
D_{s}=C_{2}(L)^{m 2},
\end{gathered}
$$

where $D_{s}$ is the dispersivity, and $C_{1}$ and $C_{2}$ are given in Tables 6.5 and 6.6 , respectively, along with $m 1$ and $m 2$ values. R-square values for equations (6.6.1) and (6.6.2) are 0.96 and 0.94 , respectively.

\begin{tabular}{ccccccc}
\hline \multirow{2}{*}{$\sigma^{2}$} & \multicolumn{5}{c}{ Dispersivity } \\
\cline { 2 - 7 } & $\mathrm{L}=1$ & $\mathrm{~L}=5$ & $\mathrm{~L}=10$ & $\mathrm{~L}=50$ & $\mathrm{~L}=100$ & $\mathrm{~L}=500$ \\
\hline 0.0001 & 0.0498 & 0.0500 & 0.0497 & 0.0498 & 0.0507 & 0.0686 \\
0.001 & 0.0498 & 0.0499 & 0.0495 & 0.0477 & 0.0639 & 0.4982 \\
0.01 & 0.0492 & 0.0510 & 0.0511 & 0.1642 & 0.5073 & 4.0672 \\
0.1 & 0.0449 & 0.0592 & 0.1372 & 0.9309 & 2.9601 & 28.6151 \\
0.25 & 0.0451 & 0.1123 & 0.2391 & 2.5441 & 6.1225 & 40.5301 \\
0.4 & 0.0573 & 0.1340 & 0.3413 & 3.4365 & 8.1834 & 48.7567 \\
0.6 & 0.0784 & 0.1824 & 0.4619 & 4.9440 & 10.9837 & 64.7589 \\
0.8 & 0.0958 & 0.1987 & 0.7057 & 6.6800 & 14.9122 & 82.4423 \\
1 & 0.1247 & 0.2159 & 0.8102 & 8.9878 & 19.9003 & 112.5246 \\
\cline { 2 - 7 } & $\mathrm{L}=1000$ & $\mathrm{~L}=2000$ & $\mathrm{~L}=4000$ & $\mathrm{~L}=6000$ & $\mathrm{~L}=8000$ & $\mathrm{~L}=10000$ \\
\cline { 2 - 7 } 0.0001 & 0.2697 & 0.7964 & 2.0630 & 4.1138 & 5.9939 & 8.1065 \\
0.001 & 2.5154 & 7.2616 & 20.5460 & 32.6517 & 45.9978 & 69.0446 \\
0.01 & 12.6500 & 30.0361 & 81.0270 & 155.2103 & 231.3154 & 324.3036 \\
0.1 & 70.0564 & 156.8923 & 333.6665 & 523.4295 & 708.0212 & 903.6889 \\
0.25 & 87.8303 & 185.7131 & 381.1019 & 569.9892 & 766.8287 & 978.5914 \\
0.4 & 101.1441 & 203.0552 & 425.5467 & 625.6709 & 866.0189 & 1061.9651 \\
0.6 & 131.0882 & 259.4990 & 528.0956 & 828.7496 & 1079.0040 & 1355.8468 \\
0.8 & 173.1833 & 344.9935 & 691.7747 & 1070.9582 & 1399.5126 & 1771.3449 \\
1 & 227.3204 & 453.3977 & 925.0844 & 1396.8663 & 1864.1378 & 2337.5588 \\
\hline
\end{tabular}

Table 6.3. Longitudinal dispersivities (mean) for the range of $L$ from $1 \mathrm{~m}$ to $10^{4} \mathrm{~m}$ under the boundary condition A 


\begin{tabular}{cccccccc}
\hline \multirow{2}{*}{$\sigma^{2}$} & \multicolumn{7}{c}{ Dispersivity } \\
\cline { 2 - 8 } & $\mathrm{L}=1$ & $\mathrm{~L}=5$ & $\mathrm{~L}=10$ & $\mathrm{~L}=50$ & $\mathrm{~L}=100$ & $\mathrm{~L}=500$ & $\mathrm{~L}=1000$ \\
\hline 0.0001 & 0.0498 & 0.0500 & 0.0497 & 0.0498 & 0.0507 & 0.0686 & 0.1426 \\
0.001 & 0.0498 & 0.0499 & 0.0495 & 0.0477 & 0.0639 & 0.4982 & 1.4690 \\
0.01 & 0.0492 & 0.0510 & 0.0511 & 0.1642 & 0.5073 & 4.0672 & 12.0999 \\
0.1 & 0.0449 & 0.0592 & 0.1372 & 0.9309 & 2.9601 & 28.6151 & 69.2489 \\
0.25 & 0.0451 & 0.1123 & 0.2391 & 2.5441 & 6.1225 & 40.5301 & 87.0760 \\
0.4 & 0.0573 & 0.1340 & 0.3413 & 3.4365 & 8.1834 & 48.7567 & 100.6075 \\
0.6 & 0.0784 & 0.1824 & 0.4619 & 4.9440 & 10.9837 & 64.7589 & 132.1320 \\
0.8 & 0.0958 & 0.1987 & 0.7057 & 6.6800 & 14.9122 & 82.4423 & 173.1823 \\
1 & 0.1247 & 0.2159 & 0.8102 & 8.9878 & 19.9003 & 112.5246 & 221.6737 \\
\hline
\end{tabular}

Table 6.4. Longitudinal dispersivities (mean) for the range of $L$ from $1 \mathrm{~m}$ to $10^{8} \mathrm{~m}$ under the boundary condition B

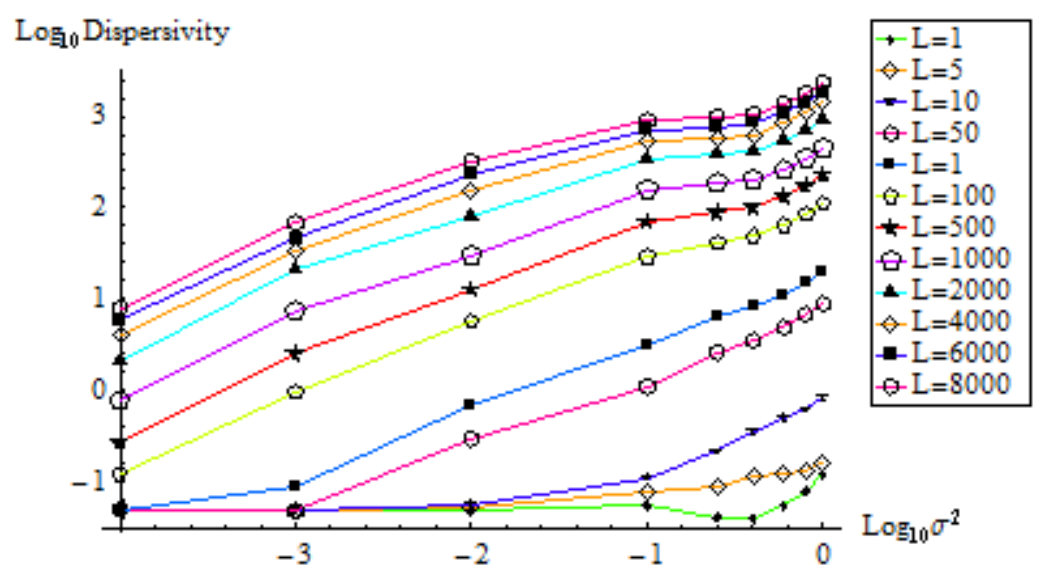

Figure 6.6. The linear relationship of Log10 (Dispersivity) vs Log10 $\left(\sigma^{2}\right)$ for different values of L. 


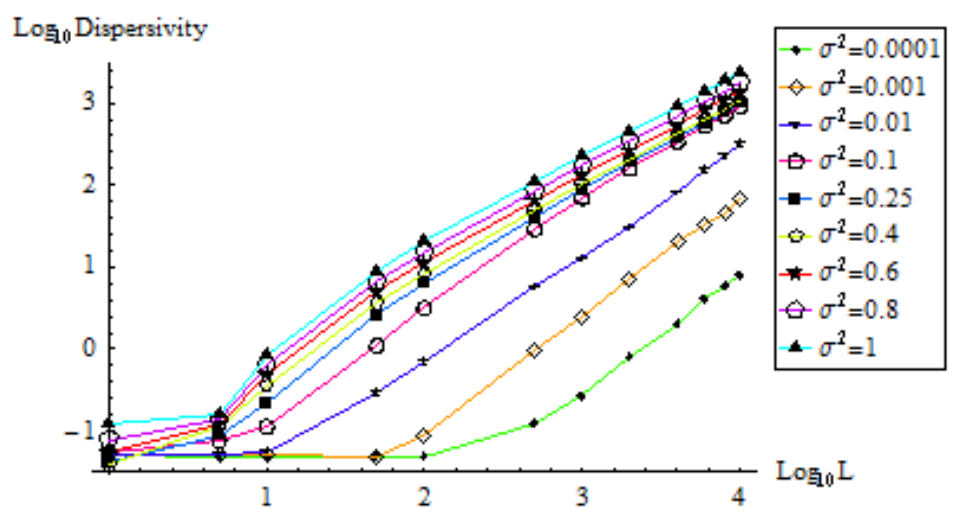

Figure 6.7. The linear relationship of Log10 (Dispersivity) vs Log10 $(L)$ for different values of $\sigma^{2}$.

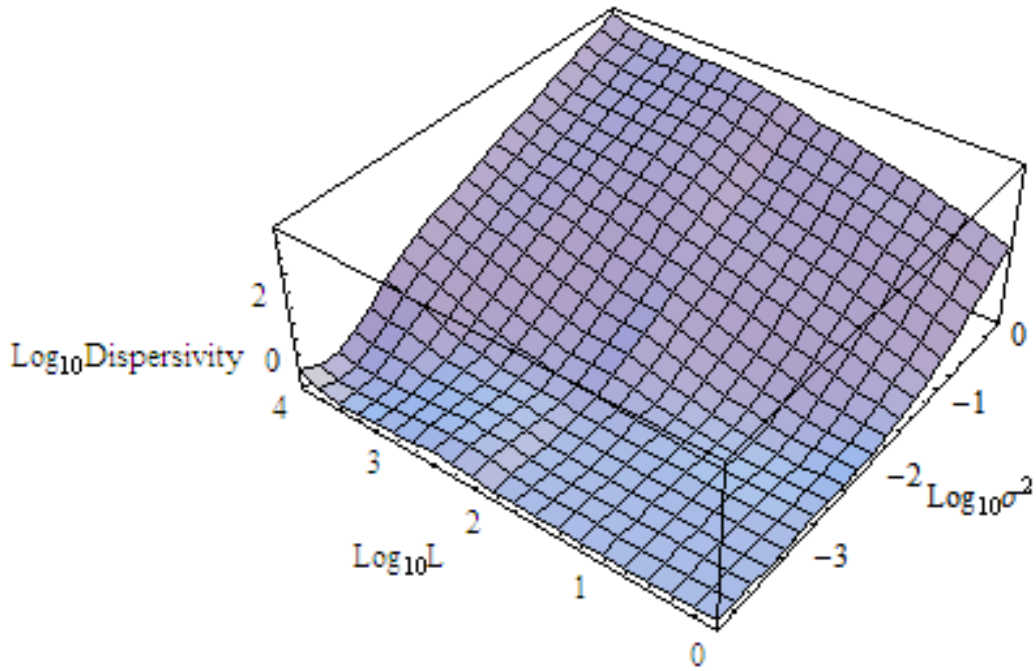

Figure 6.8. The plot of Log10 (Dispersivity) vs $\log 10\left(\sigma^{2}\right)$ and $\log 10(L)$

\begin{tabular}{c|cccccc}
\hline$L(\mathrm{~m})$ & $\mathbf{1}$ & $\mathbf{5}$ & $\mathbf{1 0}$ & $\mathbf{5 0}$ & $\mathbf{1 0 0}$ & $\mathbf{5 0 0}$ \\
\hline $\mathrm{m} 1$ & 0.039 & 0.125 & 0.311 & 0.605 & 0.677 & 0.704 \\
$\mathrm{C}_{1}$ & 0.063 & 0.124 & 0.468 & 6.275 & 16.23 & 109.6 \\
\hline$L(\mathrm{~m})$ & $\mathbf{1 0 0 0}$ & $\mathbf{2 0 0 0}$ & $\mathbf{4 0 0 0}$ & $\mathbf{6 0 0 0}$ & $\mathbf{8 0 0 0}$ & $\mathbf{1 0 0 0 0}$ \\
\hline $\mathrm{m} 1$ & 0.690 & 0.642 & 0.605 & 0.578 & 0.567 & 0.552 \\
$\mathrm{C}_{1}$ & 229.5 & 451.3 & 912.4 & 1368.7 & 1823.1 & 2281.4 \\
\hline
\end{tabular}

Table 6.5. $m 1$ and $C_{1}$ values for different $L$ for equation (6.6.1). 


\begin{tabular}{l|ccccccccc}
\hline$\sigma^{2}$ & $\mathbf{0 . 0 0 0 1}$ & $\mathbf{0 . 0 0 1}$ & $\mathbf{0 . 0 1}$ & $\mathbf{0 . 1}$ & $\mathbf{0 . 2 5}$ & $\mathbf{0 . 4}$ & $\mathbf{0 . 6}$ & $\mathbf{0 . 8}$ & $\mathbf{1 . 0}$ \\
\hline $\mathrm{m} 2$ & 0.589 & 0.897 & 1.067 & 1.150 & 1.148 & 1.148 & 1.148 & 1.148 & 1.144 \\
$\mathrm{C}_{2}$ & 0.0122 & 0.0078 & 0.0103 & 0.0168 & 0.0242 & 0.0311 & 0.0409 & 0.0535 & 0.0725 \\
\hline
\end{tabular}

Table 6.6. $m 2$ and $C_{2}$ values for different $\sigma^{2}$ for equation (6.6.2).

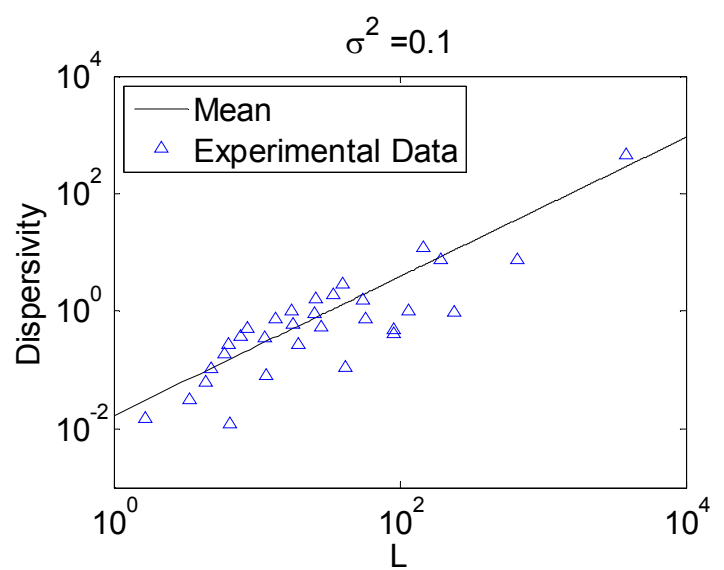

Figure 6.9. Mean dispersivity from the SSTM and experimental dispersivity vs flow length ( $\log 10$ scale).

We can estimate the approximate dispersivity values either from Figures 6.7 and 6.8, or from equations (6.6.1) and (6.6.2). It is quite logical to ask the question whether we can characterise the large scale aquifer dispersivities using a single value of $\sigma^{2}$ ? To answer this question, we resort to the published dispersivity values for aquifers. We use the dispersivity data first published by Gelhar et al. (1992) and reported to Batu (2006). We extracted the tracer tests data related to porous aquifers in 59 different locations characterised by different geologic materials. The longest flow length was less than $10000 \mathrm{~m}$. We then plotted the experimental data and overlaid the plot with the dispersivity vs $L$ curves from the SSTM for each $\sigma^{2}$ value. Figure 6.9 shows the plots, and $\sigma^{2}=0.1$ best fit to the experimental data. In other words, by using one value of $\sigma^{2}$, we can obtain the dispersivity for any length of the flow by using the SSTM. We can also assume that each experimental data point represents the mean dispersivity for any length of the flow by using the SSTM. We can also assume that each experimental data point represents the mean dispersivity at a particular flow length. If that is the case, Figure 6.9 can be interpreted as follows: by using the SSTM, we can obtain sufficiently large number of realisations for particular values of $\sigma^{2}$ and the mean flow velocity, and the mean values of the dispersivities estimated for those concentration realisations do represent the experimental dispersivities. $\sigma^{2}$ can be hypothesised to indicate the type of media (e.g. fractured, porous etc.). These findings support the hypothesis that the dimensionless SSTM is scale-independent, i.e., one value of $\sigma^{2}$ would be sufficient to characterise the dispersivity at different flow lengths. It is important to note that the role of the mean velocity in these calculations. We used $0.5 \mathrm{~m} /$ day to represent an indicative value in real aquifers, but the character of solutions do not change, if we assume a different value; only the specific values of $\sigma^{2}$ would be changed to represent a given flow situation. 


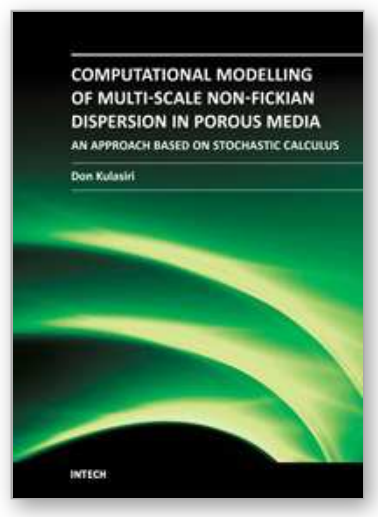

\author{
Computational Modelling of Multi-scale Solute Dispersion in \\ Porous Media - An Approach Based on Stochastic Calculus \\ Edited by
}

ISBN 978-953-307-726-0

Hard cover, 234 pages

Publisher InTech

Published online 04, November, 2011

Published in print edition November, 2011

This research monograph presents a mathematical approach based on stochastic calculus which tackles the "cutting edge" in porous media science and engineering - prediction of dispersivity from covariance of hydraulic conductivity (velocity). The problem is of extreme importance for tracer analysis, for enhanced recovery by injection of miscible gases, etc. This book explains a generalised mathematical model and effective numerical methods that may highly impact the stochastic porous media hydrodynamics. The book starts with a general overview of the problem of scale dependence of the dispersion coefficient in porous media. Then a review of pertinent topics of stochastic calculus that would be useful in the modeling in the subsequent chapters is succinctly presented. The development of a generalised stochastic solute transport model for any given velocity covariance without resorting to Fickian assumptions from laboratory scale to field scale is discussed in detail. The mathematical approaches presented here may be useful for many other problems related to chemical dispersion in porous media.

\title{
How to reference
}

In order to correctly reference this scholarly work, feel free to copy and paste the following:

Don Kulasiri (2011). Multiscale, Generalised Stochastic Solute Transport Model in One Dimension, Computational Modelling of Multi-scale Solute Dispersion in Porous Media - An Approach Based on Stochastic Calculus, (Ed.), ISBN: 978-953-307-726-0, InTech, Available from:

http://www.intechopen.com/books/computational-modelling-of-multi-scale-solute-dispersion-in-porous-mediaan-approach-based-on-stochastic-calculus/multiscale-generalised-stochastic-solute-transport-model-in-onedimension

\section{INTECH}

open science | open minds

\author{
InTech Europe \\ University Campus STeP Ri \\ Slavka Krautzeka 83/A \\ 51000 Rijeka, Croatia \\ Phone: +385 (51) 770447 \\ Fax: +385 (51) 686166 \\ www.intechopen.com
}

\author{
InTech China \\ Unit 405, Office Block, Hotel Equatorial Shanghai \\ No.65, Yan An Road (West), Shanghai, 200040, China \\ 中国上海市延安西路65号上海国际贵都大饭店办公楼 405 单元 \\ Phone: +86-21-62489820 \\ Fax: $+86-21-62489821$
}


(C) 2011 The Author(s). Licensee IntechOpen. This is an open access article distributed under the terms of the Creative Commons Attribution 3.0 License, which permits unrestricted use, distribution, and reproduction in any medium, provided the original work is properly cited. 